\title{
Association of nNOS and Rho-kinase with age-associated erectile dysfunction in Sprague-Dawley rats
}

\author{
ZHIBO DING ${ }^{1}$, XINGJIE SHEN ${ }^{2}$ and YUNFEI LI ${ }^{3}$ \\ ${ }^{1}$ Department of Urology, The Fourth People's Hospital of Jinan, Jinan, Shandong 250031; \\ ${ }^{2}$ Department of Gastroenterology, Jinan Central Hospital Affiliated to Shandong University, Jinan, Shandong 250013; \\ ${ }^{3}$ Center for Reproductive Medicine, The General Hospital of Jinan Military Command, Jinan, Shandong 250031, P.R. China
}

Received September 14, 2015; Accepted October 28, 2016

DOI: $10.3892 /$ etm.2017.4064

\begin{abstract}
The present study aimed to investigate the changes of Rho-associated kinase (Rho-kinase) and neuronal nitric oxide synthase (nNOS) expression in age-associated erectile dysfunction (ED) in Sprague-Dawley (SD) rats. A total of 100 intact male SD rats were divided into 20 groups according to their age (5-24 months; rats that were the same age in months were in the same group). Erectile response measurements were performed and the functional index intracavernosal pressure/mean arterial pressure (ICP/MAP) was tested, followed by detection of Rho-kinase and nNOS protein by western blot analysis. Finally, correlation analyses of the association between ICP/MAP and Rho-kinase, nNOS, or nNOS/Rho-kinase, as well as between age and nNOS or Rho-kinase, were performed. The functional index ICP/MAP decreased with age in SD rats. Moreover, the expression of nNOS protein decreased, while Rho-kinase expression increased, indicating that the nNOS/Rho-kinase ratio decreased with age. The Pearson's correlation coefficients for the association between ICP/MAP and Rho-kinase, nNOS and nNOS/Rho-kinase ratio were $-0.917,0.853$ and 0.937 , respectively $(\mathrm{P}<0.01)$. Furthermore, nNOS was found to be significantly negatively correlated with age $(\mathrm{r}=-0.855 ; \mathrm{P}<0.01)$, whereas Rho-kinase was positively correlated with age $(\mathrm{r}=0.943 ; \mathrm{P}<0.01)$. Age-associated ED was therefore correlated with decreased nNOS and increased Rho-kinase, indicating that the nNOS/Rho-kinase ratio may be used as a candidate indicator of age-associated ED.
\end{abstract}

\section{Introduction}

Erectile dysfunction (ED) is a prevalent health problem worldwide and its incidence is rising $(1,2)$. The clinical

Correspondence to: Dr Yunfei Li, Center for Reproductive Medicine, The General Hospital of Jinan Military Command, 25 Shifan Road, Jinan, Shandong 250031, P.R. China

E-mail: licloudf@163.com

Key words: erectile dysfunction, correlation coefficient, neuronal nitric oxide synthase, Rho-associated kinase, intracavernosal pressure/mean arterial pressure features of ED are strongly associated with age; although a number of techniques have been used to treat ED (3-5), the incidence rates of ED in men aged $<59$ years, $60-69$ years and $>69$ years are 12, 22 and $30 \%$, respectively $(2,6)$. ED not only has a significant impact on psychosocial and physical health, but also affects the life quality of patients and their partners. Furthermore, ED is an emerging risk marker for cardiovascular disease, including ischemic heart disease, heart failure and peripheral vascular disease (7). Therefore, investigating the molecular mechanisms of age-associated ED may facilitate the prevention of disease progression and the development of novel therapies for treatment of this disease.

ED is characterized by the persistent inability to attain or maintain a penile erection sufficient to permit satisfactory sexual intercourse (6). Numerous factors have been found to be closely associated with erectile function. For instance, neuronal nitric oxide synthase (nNOS) has been shown to regulate the recovery of erectile function following partial cavernous nerve injury and exerts a suppressive role by inducing apoptotic change in penile tissue (8). Hurt et al (9) also reported that cyclic AMP-dependent phosphorylation of nNOS mediated the initiation as well as the maintenance of penile erection. In addition, the expression of Rho-associated kinase (Rho-kinase) was found to be increased in the penises of aged rats (10) and it has been demonstrated that inhibiting Rho-kinase protein improves erectile function in aging rats (11). Previous studies have confirmed that inhibition of Rho-kinase in a rat model of cavernous nerve injury decreases penile cell apoptosis and prevents the development of ED $(12,13)$. Although it has been observed that the expression of nNOS protein is decreased in the penis of aged Sprague-Dawley (SD) rats (14), the association between decreased nNOS and increased Rho-kinase levels with age-associated ED in SD rats has not been fully investigated.

To address this issue, in the current study, the expression of Rho-kinase and nNOS was assessed and the erectile functional index intracavernosal pressure/mean arterial pressure (ICP/MAP) was determined in SD rats of different ages (5-24 months). A correlation analysis was performed to evaluate the association between age and Rho-kinase or nNOS, and between ICP/MAP and Rho-kinase, nNOS or nNOS/Rho-kinase. The present study aimed to determine the changes in Rho-kinase and nNOS expression in aged-associated $\mathrm{ED}$ in $\mathrm{SD}$ rats, as well as elucidate their regulatory 
mechanisms. The findings of the present study may provide a theoretical basis for drug therapy of age-associated ED.

\section{Materials and methods}

Animals. A total of 100 intact male SD rats (age, 5-24 months; weight, 325-375 g) were obtained from the Experimental Center of Jinan Clinical Institute, Medical College of Taishan (Taishan, China). Rats were divided into 20 groups $(n=5$ in each) according to their age (5-24 months; rats that were the same age in months were put in the same group). All rats were housed at a constant temperature $\left(23 \pm 1^{\circ} \mathrm{C}\right)$ and humidity $(50 \pm 5 \%)$ with free access to food and tap water under a $12-\mathrm{h}$ light/dark cycle prior to the experiments. The experimental protocol was in accordance with the 'Guiding Principles in the Care and Use of Animals' endorsed by the State Department of China. The present study was approved by the Ethics Committee and Animal Management Committee of the Fourth People's Hospital of Jinan (Jinan, China).

Erectile response measurements. Rats were anesthetized by intraperitoneal injection of $10 \%$ chloral hydrate $(0.3 \mathrm{ml} / 100 \mathrm{~g}$ body weight, Sigma-Aldrich; Merck Millipore, Darmstadt, Germany). The experimental animals were fixed on a console. Following neck disinfection, the skin was incised near the trachea and thyroid cartilage to expose the neck muscles. The carotid artery was separated along the trachea, below the thyroid cartilage. The carotid artery was then pulled out using a 4-gauge wire and cannulated by insertion of a 25-gauge needle. Subsequently, the carotid artery was fixed and connected to a pressure transducer (Gould Electronics, Cleveland, OH, USA) for continuous monitoring of the MAP. In addition, 5-10 mm from the distal section of the rat penis, the corpus cavernosum was cannulated by insertion of a 25 -gauge needle and then connected to a pressure transducer for continuous monitoring of ICP. Heparinised saline (100 U/ml, Sigma-Aldrich; Merck Millipore) was added into a connection pipe between the needle and pressure transducer to prevent coagulation. The abdominal cavity was then opened, exposing the major pelvic ganglion (MPG), on which a platinum bipolar electrode was positioned. During stimulation, the position of the electrode was adjusted until a maximal voltage-induced response was achieved. All experimental rats were given a 5-V stimulus and the MAP, ICP and ICP/MAP were recorded (15).

Rho-kinase and nNOS protein detection. The expression levels of Rho-kinase and nNOS proteins in the corpus cavernosum of rats of different ages were detected by western blot analysis as described previously (14), with certain modifications. Following blocking with $5 \%$ skimmed milk, the membrane was incubated with primary antibodies, including rabbit anti-rat Rho-kinase (1:1,000, orb312940, Shanghai Qifa Bio-technology Ltd., Shanghai, China), rabbit anti-rat nNOS (1:1,000, SRP08644b; Tianjin Saier Bio-technology Ltd., Tianjin, China) and GAPDH polyclonal antibody (1:5,000, ABP57259; Wuhan Amyjet Scientific Inc., Wuhan, China) overnight at $4^{\circ} \mathrm{C}$. Subsequently, the membranes were incubated with goat anti-rabbit horseradish peroxidase-linked secondary antibody (1:5,000, A21020; Wuhan Amyjet Scientific Inc.) at $37^{\circ} \mathrm{C}$ for $2 \mathrm{~h}$, followed by enhanced chemiluminescence with diaminobenzidine tetrahydrochloride substrate (Pierce; Thermo Fisher Scientific, Inc., Waltham, MA, USA) in the dark. GAPDH served as a loading control. The band intensity was then quantified using a computerized image analysis system (16). The expression levels of Rho-kinase and nNOS protein were presented as the ratio of Rho-kinase and nNOS protein normalized to GAPDH.

Correlation analysis. Pearson's correlation analyses between ICP/MAP and Rho-kinase, ICP/MAP and nNOS as well as between ICP/MAP and nNOS/Rho-kinase were performed. In addition, the correlation between the age of experimental rats and Rho-kinase as well as nNOS was assessed.

Statistical analysis. The data obtained from multiple experiments were presented as the mean \pm standard deviation. Pearson's correlation analysis was performed to assess the correlation between ICP/MAP, nNOS, Rho-kinase and nNOS/Rho-kinase. All statistical analyses were performed using SPSS 16.0 (SPSS, Inc., Chicago, IL, USA). A two-sided $t$ test was performed to analyze significant differences among groups. $\mathrm{P}<0.05$ was considered to indicate a statistically significant difference.

\section{Results}

Penile erectile functional measurements of SD rats of different ages. During the animal experiment, in 5 groups, one out of the 5 rats in each group succumbed. As a result, by the end of the experiment, 5 groups only consisted of 4 rats, while in the other 15 groups there were 5 rats in each group. Analysis of the penile erectile functional measurements of all SD rats of different ages (5-24 months) showed that the baseline ICP increased with age and was $8.2 \pm 4.3 \mathrm{mmHg}$, and the baseline MAP was $13.8 \pm 5.7 \mathrm{mmHg}$ (data not shown). As shown in Fig. 1, the penile erectile functional index, ICP/MAP, decreased with age. For instance, ICP/MAP was $0.85 \pm 0.04$ at 5 months, $0.62 \pm 0.05$ at 15 months and $0.46 \pm 0.05$ at 24 months, confirming that penile erectile function decreased with age. In addition, levels of the nNOS protein markedly decreased with age $(1.65 \pm 0.04,1.23 \pm 0.06$ and $1.19 \pm 0.06$ at 5,15 and 24 months, respectively), while levels of the Rho-kinase protein increased with age $(1.59 \pm 0.03,1.85 \pm 0.04$ and $2.95 \pm 0.04$ at 5 , 12 and 24 months, respectively). Notably, the nNOS/Rho-kinase ratio decreased with age $(1.02 \pm 0.04,0.63 \pm 0.05$ and $0.37 \pm 0.04$ at 5, 12 and 24 months, respectively). Statistical analysis indicated that significant differences existed between groups of different age rats, and that the ICP/MAP ratio, nNOS levels and the $\mathrm{nNOS} / \mathrm{Rho}$-kinase ratio were decreased, while levels of Rho-kinase increased with age $(\mathrm{P}<0.05$, Table I).

Correlation analysis. A correlation analysis was performed to investigate whether levels of Rho-kinase and nNOS were associated with the erectile function of SD rats, as well as to validate the association between age and nNOS or Rho-kinase. As presented in Table II, the correlation analysis revealed that Rho-kinase was negatively correlated with ICP/MAP ratio (Pearson's $r=-0.917 ; \mathrm{P}<0.01$ ). Moreover, nNOS was positively correlated with the ICP/MAP ratio (Pearson's $\mathrm{r}=0.853 ; \mathrm{P}<0.01)$. Similarly, the $\mathrm{nNOS} / \mathrm{Rho}$-kinase ratio was also positively correlated with the ICP/MAP ratio (Pearson's 
Table I. Analysis of the differences of penile erectile functional indexes between groups of different age using t-test.

\begin{tabular}{lccc}
\hline $\begin{array}{l}\text { Penile erectile } \\
\text { functional indexes }\end{array}$ & Age, months & T value & P-value \\
\hline ICP/MAP ratio & 5 vs. 15 & 7.172 & $\mathrm{P}=0.002$ \\
& 15 vs. 24 & 4.545 & $\mathrm{P}=0.010^{\mathrm{a}}$ \\
nNOS & 5 vs. 15 & $\mathrm{t}=11.366$ & $\mathrm{P}<0.001^{\mathrm{a}}$ \\
& 15 vs. 24 & $\mathrm{t}=0.889$ & $\mathrm{P}=0.424$ \\
nNOS/Rho-kinase & 5 vs. 15 & $\mathrm{t}=11.302$ & $\mathrm{P}<0.001^{\mathrm{a}}$ \\
ratio & 15 vs. 24 & $\mathrm{t}=7.576$ & $\mathrm{P}=0.002^{\mathrm{a}}$ \\
Rho-kinase & 5 vs. 15 & $\mathrm{t}=9.875$ & $\mathrm{P}=0.001^{\mathrm{a}}$ \\
& 15 mo vs. $24 \mathrm{mo}$ & $\mathrm{t}=35.585$ & $\mathrm{P}<0.001^{\mathrm{a}}$ \\
\end{tabular}

${ }^{a} \mathrm{P}<0.05$, indicating a significant difference. nNOS, neuronal nitric oxide synthase; ICP/MAP, intracavernosal pressure/mean arterial pressure; Rho-kinase, Rho-associated kinase.

Table II. Correlation analysis.

\begin{tabular}{lcc}
\hline Comparison & Pearson's r & P-value \\
\hline Rho-kinase vs. ICP/MAP ratio & -0.917 & $\mathrm{P}<0.01$ \\
nNOS vs. ICP/MAP ratio & 0.853 & $\mathrm{P}<0.01$ \\
nNOS/Rho-kinase ratio vs. & 0.937 & $\mathrm{P}<0.01$ \\
ICP/MAP ratio & & \\
nNOS vs. Age & -0.855 & $\mathrm{P}<0.01$ \\
Rho-kinase vs. Age & 0.943 & $\mathrm{P}<0.01$ \\
\hline
\end{tabular}

nNOS, neuronal nitric oxide synthase; ICP/MAP, intracavernosal pressure/mean arterial pressure; Rho-kinase, Rho-associated kinase.

$\mathrm{r}=0.937 ; \mathrm{P}<0.01)$. In addition, the correlation analysis showed that nNOS was significantly negatively correlated with age (Pearson's r=-0.855; $\mathrm{P}<0.01$ ), while Rho-kinase was positively correlated with age (Pearson's $\mathrm{r}=0.943 ; \mathrm{P}<0.01)$.

\section{Discussion}

Physiological aging is a significant risk factor for ED. The present study investigated the mechanisms of age-associated ED by assessing changes in erectile function as well as the ICP/MAP ratio, nNOS and Rho-kinase protein levels in SD rats. The results indicated that the levels of nNOS protein and the ICP/MAP ratio decreased with age, while levels of Rho-kinase protein increased with age. Furthermore, the study also demonstrated that the ICP/MAP ratio was positively correlated with $\mathrm{nNOS}$ and the $\mathrm{nNOS} / \mathrm{Rho-kinase}$ ratio and negatively correlated with Rho-kinase.

A previous study found that older rats have decreased relative $\mathrm{nNOS}$ protein levels compared with younger rats $(1 \pm 0.15$ vs. $1.7 \pm 0.15$ ) (14). Another study demonstrated that there is $\sim 13 \%$ decrease in the gene expression of nNOS in the penises of aged rats (17). In addition, increasing evidence has shown that endothelial and neuronal-derived nitric oxide acts as an important

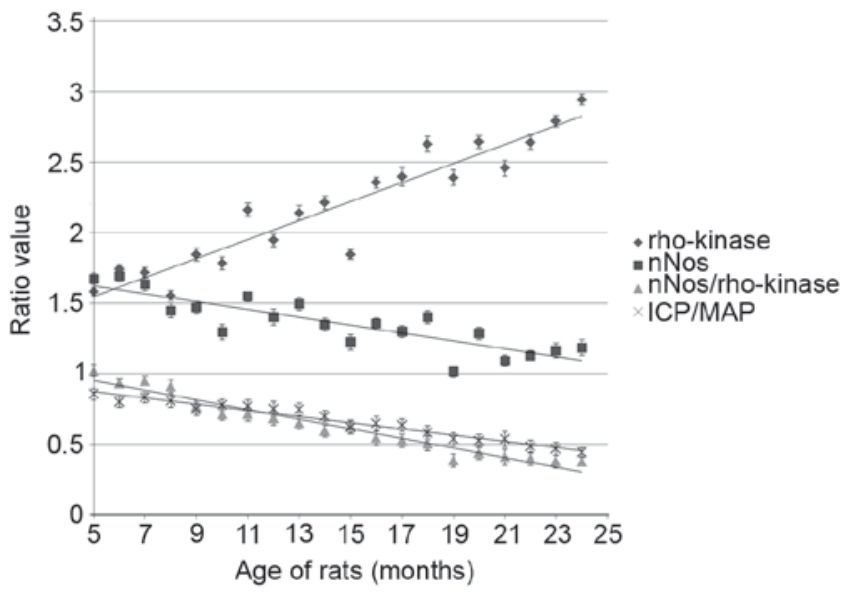

Figure 1. Penile erectile functional measurements of ICP/MAP ratio, nNOS, Rho-kinase and nNOS/Rho-kinase ratio of Sprague-Dawley rats of different age. The protein expression of nNOS and Rho-Kinase was normalized to GAPDH. nNOS, neuronal nitric oxide synthase; ICP/MAP, intracavernosal pressure/mean arterial pressure; Rho-kinase, Rho-associated kinase.

mediator of corpus cavernosal smooth-muscle relaxation and penile erection in rats with ED (18). nNOS was also shown to be involved in mediating initiation as well as maintenance of penile erection via cyclic adenosine monophosphate-dependent phosphorylation (9). Furthermore, changes in the dimethylarginine dimethylaminohydrolase/asymmetric dimethylarginine/NOS pathway were found to accompany lower ICP in an older group of rats (18 months old), compared with that of a younger group (3 months old) (19). The ICP/MAP ratio is the common functional index to determine erectile function (10). Qiu et al (20) also demonstrated that immediate and delayed treatment with stromal vascular fraction resulted in a significantly increased ICP/MAP ratio and increased expression of nNOS compared with the vehicle-treated group, implying that increased expression of nNOS may enhance recovery of erectile function in a rat model of cavernous nerve injury via increasing the ICP/MAP ratio. In the present study, decreased expression of nNOS was found in aged rats and the ICP/MAP ratio decreased with age. Additionally, the expression of nNOS was positively correlated with the ICP/MAP ratio $(r=0.853)$. Therefore, the results of the present study are in line with previous findings and imply that the decreased erectile function of SD rats is associated with increasing age and that decreased nNOS may be a reason for age-associated ED.

Furthermore, accumulating evidence has demonstrated that age-associated ED is likely to be associated with an increased Rho-kinase activity and antagonism of Rho-kinase activity can attenuate the age-associated decline in male erectile function (11). Rho-kinase was found to affect the erectile response via enhancing the vasoconstriction state of the penile smooth muscle in lean and obese-diabetic Zucker rats (21). Rho-kinase may have an important role in maintaining penile detumescence and vasoconstriction of the corporal vasculature (22). Meanwhile, increased RhoA/Rho-kinase signaling activation is involved in impaired erectile function during the ageing process (10). A study by Gao et al (14) confirmed that the expression of Rho-kinase protein was increased in old rats (age, 20-22 months) compared to that in young rats (age, 6-9 months). Furthermore, inhibition of Rho-kinase by a selective Rho kinase 
inhibitor, Y-27632, was reported to increase ICP in a rat model of erection, thus preventing the development of vasculogenic $\mathrm{ED}$ (23). An increase in the ICP/MAP ratio in response to electrical stimulation of the MPG has been shown to be blocked by inhibitors of nitric oxide production or signaling, which is in line with the hypothesis that nitric oxide induces penile erection via inhibition of RhoA/Rho-kinase signaling (24). The present study found a negative correlation between Rho-kinase and age. Furthermore, Rho-kinase was negatively associated with the ICP/MAP ratio ( $r=-0.917)$. All of these findings suggested that increased Rho-kinase may contribute to age-associated ED in $\mathrm{SD}$ rats.

Of note, intracavernosal gene therapy with penile nNOS has been shown to correct age-associated ED (25). High expression of RhoA was also shown to result in a flaccid state of the corpus cavernosum by RhoA-mediated $\mathrm{Ca}^{2+}$ sensitization, which was activated by a Rho-kinase inhibitor suitable for the treatment of ED (26). In addition, the ICP/MAP ratio is the common functional index to determine erectile function (10). The present study found that the nNOS/Rho-kinase ratio decreased with age and was positively correlated with the ICP/MAP ratio $(\mathrm{r}=0.937$, $\mathrm{P}<0.01$ ), suggesting that a decreased $\mathrm{nNOS} /$ Rho-kinase ratio may be a cause of age-associated ED and the nNOS/Rho-kinase ratio may serve as an alternative to the ICP/MAP ratio as a promising indicator of erectile function.

The present study had certain limitations. First, there were only 5 rats in each of the 20 groups according to their age (5-24 months). Theoretically, the rats aged 5-10 months were considered young compared to rats aged 15-24 months. Although there were only 5 rats in each age group, the sample size was justified for exploring the effect of age on the association of ED with nNOS and Rho-kinase expression; however, it is required to verify the results of the present study by further studies using a larger number of rats in each group.

In conclusion, the results of the present study indicated that decreased nNOS and increased Rho-kinase expression may be important reasons for age-associated ED. Moreover, the nNOS/Rho-kinase ratio may be an alternative to the ICP/MAP ratio to serve as a possible indicator of erectile function. In addition, increasing nNOS and decreasing Rho-kinase expression may represent potential therapeutic strategies for the treatment of ED.

\section{References}

1. Cai L, Jiang M,Zeng M, Xing W, Wen Y and Zhang B: Age-specific clinical features of erectile dysfunction. Health 6,2014.

2. Bacon CG, Mittleman MA, Kawachi I, Giovannucci E, Glasser DB and Rimm EB: Sexual function in men older than 50 years of age: Results from the health professionals follow-up study. Ann Intern Med 139: 161-168, 2003.

3. Steers WD: Pharmacologic treatment of erectile dysfunction. Rev Urol 4 (Suppl 3): S17-S25, 2002.

4. de Andrade E, de Mesquita AA, Claro Jde A, de Andrade PM, Ortiz V, Paranhos M and Srougi M: Study of the efficacy of Korean Red Ginseng in the treatment of erectile dysfunction. Asian J Androl 9: 241-244, 2007.

5. Shabsigh R, Rajfer J, Aversa A, Traish AM, Yassin A, Kalinchenko SY and Buvat J: The evolving role of testosterone in the treatment of erectile dysfunction. Int J Clin Pract 60: 1087-1092, 2006.

6. Montorsi F, Adaikan G, Becher E, Giuliano F, Khoury S, Lue TF, Sharlip I, Althof SE, Andersson KE, Brock G, et al: Summary of the recommendations on sexual dysfunctions in men. J Sex Med 7: $3572-3588,2010$
7. Inman BA, St Sauver JL, Jacobson DJ, McGree ME, Nehra A, Lieber MM, Roger VL and Jacobsen SJ: A population-based, longitudinal study of erectile dysfunction and future coronary artery disease. Mayo Clin Proc 84: 108-113, 2009.

8. Sezen SF, Lagoda G and Burnett AL: Neuronal nitric oxide signaling regulates erection recovery after cavernous nerve injury. J Urol 187: 757-763, 2012.

9. Hurt KJ, Sezen SF, Lagoda GF, Musicki B, Rameau GA, Snyder SH and Burnett AL: Cyclic AMP-dependent phosphorylation of neuronal nitric oxide synthase mediates penile erection. Proc Natl Acad Sci 109: 16624-16629, 2012.

10. Jin L, Liu T, Lagoda GA, Champion HC, Bivalacqua TJ and Burnett AL: Elevated RhoA/Rho-kinase activity in the aged rat penis: Mechanism for age-associated erectile dysfunction. FASEB J 20: 536-538, 2006.

11. Rajasekaran M, White S, Baquir A and Wilkes N: Rho-kinase inhibition improves erectile function in aging male brown-norway rats. J Androl 26: 182-188, 2005.

12. Hannan JL, Albersen M, Kutlu O, Gratzke C, Stief CG, Burnett AL, Lysiak JJ, Hedlund P and Bivalacqua TJ: Inhibition of Rho-kinase improves erectile function, increases nitric oxide signaling and decreases penile apoptosis in a rat model of cavernous nerve injury. J Urol 189: 1155-1161, 2013.

13. Cho MC, Park K, Kim SW and Paick JS: Restoration of erectile function by suppression of corporal apoptosis, fibrosis and corporal veno-occlusive dysfunction with Rho-kinase inhibitors in a rat model of cavernous nerve injury. J Urol 193: 1716-1723, 2015.

14. Gao BH, Zhao ST, Meng FW, Shi BK, Liu YQ and Xu ZS: Y-27632 improves the erectile dysfunction with ageing in SD rats through adjusting the imbalance between nNo and the Rho-kinase pathways. Andrologia 39: 146-150, 2007.

15. Mills TM, Chitaley K, Wingard CJ, Lewis RW and Webb RC: Effect of Rho-kinase inhibition on vasoconstriction in the penile circulation. J Appl Physiol (1985) 91: 1269-1273, 2001.

16. Bivalacqua TJ, Champion HC, Usta MF, Cellek S, Chitaley K, Webb RC, Lewis RL, Mills TM, Hellstrom WJ and Kadowitz PJ: RhoA/Rho-kinase suppresses endothelial nitric oxide synthase in the penis: A mechanism for diabetes-associated erectile dysfunction. Proc Natl Acad Sci USA 101: 9121-9126, 2004.

17. Rajasekaran M, Kasyan A, Jain A, Kim SW and Monga M: Altered growth factor expression in the aging penis: The Brown-Norway rat model. J Androl 23: 393-399, 2002.

18. Cartledge JJ, Minhas S, Eardley I and Morrison JF: Endothelial and neuronal-derived nitric oxide mediated relaxation of corpus cavernosal smooth muscle in a rat, in vitro, model of erectile function. Int J Impot Res 12: 213-221, 2000.

19. Wang JH, Chen D, Zhang KQ, Zhang $\mathrm{H}$ and Fu Q: Effect of DDAH/ADMA/NOS regulation pathway on cavernae corporum cavernosorum rat penis of different age. Andrologia 48: 262-267, 2015.

20. Qiu X, Fandel TM, Ferretti L, Albersen M, Orabi H, Zhang H, Lin G, Lin CS, Schroeder T and Lue TF: Both immediate and delayed intracavernous injection of autologous adipose-derived stromal vascular fraction enhances recovery of erectile function in a rat model of cavernous nerve injury. Eur Urol 62: 720-727, 2012.

21. Wingard C, Fulton D and Husain S: Altered penile vascular reactivity and erection in the zucker obese-diabetic rat. J Sex Med 4: 348-363, 2007.

22. Chitaley K, Wingard CJ, Clinton Webb R, Branam H, Stopper VS, Lewis RW and Mills TM: Antagonism of Rho-kinase stimulates rat penile erection via a nitric oxide-independent pathway. Nat Med 7: 119-122, 2001.

23. Park K, Kim SW, Rhu KS and Paick JS: Chronic administration of an oral Rho kinase inhibitor prevents the development of vasculogenic erectile dysfunction in a rat model. J Sex Med 3: 996-1003, 2006.

24. Chitaley K, Bivalacqua TJ, Champion HC, Usta MF, Hellstrom WJ, Mills TM and Webb RC: Adeno-associated viral gene transfer of dominant negative RhoA enhances erectile function in rats. Biochem Biophys Res Commun 298: 427-432, 2002.

25. Magee TR, Ferrini M, Garban HJ, Vernet D, Mitani K, Rajfer J and Gonzalez-Cadavid NF: Gene therapy of erectile dysfunction in the rat with penile neuronal nitric oxide synthase. Biol Reprod 67: 20-28, 2002.

26. Wang H, Eto M, Steers WD, Somlyo AP and Somlyo AV: RhoA-mediated $\mathrm{Ca} 2+$ sensitization in erectile function. J Biol Chem 277: 30614-30621, 2002. 